\title{
Percepción de calidad de vida en trabajodores víctimas de accidente laboral que terminó en amputación
}

\author{
Luisa Fernanda Yasnó-Varila* \\ Diego Fernando Correa-Echeverry** \\ Iván Dario Morales-Correa*** \\ Martha Cecilia Veloza-Morales****
}

Recibido en 12 de junio de 2019, aceptado en 26 de agosto de 2020

\section{Citar este artículo así:}

Yasnó-Varila LF, Correa-Echeverry DF, Morales-Correa ID, Veloza-Morales MC. Percepción de calidad de vida en trabajadores víctimas de accidente laboral que terminó en amputación. Hacia. Promoc. Salud. 2021; 26 (1): 2336 DOI: $10.17151 / \mathrm{hpsal} .2021 .26 .1 .4$

Objetivo: describir la percepción de calidad de vida de las personas que han sufrido amputación por un accidente laboral. Metodología: investigación cualitativa con la aplicación de entrevista semiestructurada de 10 preguntas abiertas a seis trabajadores que sufrieron un accidente laboral que terminó en amputación. La información fue recolectada, analizada y clasificada de forma manual en categorías emergentes. Resultados: los participantes son de sexo masculino, de 34 y 52 años de edad, su domicilio son los municipios del eje cafetero y norte del valle, el estado civil es 5 casados y 1 separado, el nivel educativo 2 en primaria, 3 con secundaria y 1 técnico profesional. Todos afiliados al sistema de salud. Los segmentos corporales amputados son: 3 amputados del Miembro Superior, los otros tres amputados del Miembro Inferior. Conclusiones: su calidad de vida según las capacidades funcionales humanas centrales definidas por Nussbaum reflejan que posterior al siniestro fue afectada en la pérdida seguridad y confianza en sí mismos, impidiendo las actividades de tipo social. Su condición actual los limita en mejorar sus ingresos por su condición de discapacidad. El dolor crónico y la desadaptación protésica son comunes. Al igual que el uso de aditamentos y las dificultades en el traslado. Es evidente el fortalecimiento de la red de apoyo familiar primaria, sin embargo, al finalizar su rehabilitación hay desinterés en actividades sociales. Los participantes tienen proyectos a futuro concretos que involucran su familia. Finalmente, su proceso de rehabilitación se limitó a lo básico y no contaron con otro tipo de apoyo.

\section{Palabros clave}

Percepción, amputación, accidente de trabajo, calidad de vida, trabajador (Fuente: DeCS, BIREME).

\footnotetext{
* Maestrante en Salud Pública y Desarrollo Social. Especialista en Gerencia de la Calidad de los Servicios de Salud. Especialista en Seguridad y Salud en el Trabajo. Especialista en Auditoría en Salud. Fisioterapeuta. Estudiante. Bogotá. Correo Electrónico: 1fyasno@gmail.com

(D) orcid.org/0000-0002-2592-1485. Google

** Maestrante en Salud Pública y Desarrollo Social. Especialista en Rehabilitación Cardiopulmonar. Especialista en Salud Ocupacional, Gerencia y Control de Riesgos. Especialista en Epidemiología. Especialista en Auditoría en Salud. Especialización de Instituciones de Salud. Médico. Estudiante. Bogotá. Correo Electrónico: diegocorrea50@hotmail.com (D) orcid.org/0000-0001-8467-2960. Google

${ }^{* * *}$ Maestrante en Salud Pública y Desarrollo Social. Especialista en Auditoría en Salud. Especialista en Gerencia en Instituciones de Salud. Enfermero. Estudiante. Bogotá. Correo electrónico: jefe.ivan@gmail.com. (D) orcid.org/0000-0002-7512-8442. Google

*** Magíster Desarrollo Educativo y Social. Magíster en Enfermería-Salud Familiar. Especialista en Docencia Universitaria. Enfermera. Docente Asesora. Bogotá. Correo Electrónico: mveloza@areandina.edu.co (D) orcid.org/0000-0002-4433-9263. Google
} 


\title{
Perception of quality of life in workers who are victims of a work accident that ended in amputation.
}

\begin{abstract}
Objective: to describe the perception of quality of life of people who have suffered amputation due to a work accident. Methodology: qualitative research with the application of a 10 open questions semi-structured interview to six workers who had suffered an occupational accident that ended in amputation. The information was collected, analyzed and classified manually in emerging categories. Results: the participants are 34 and 52 years old males from the municipalities of the coffee triangle area and north of the Department of Valle del Cauca. Their marital status is 5 married and 1 separated, their educational level is 2 primary school, 3 secondary school and 1 professional technician. All of them are affiliated to the health system. The body segments amputated are: 3 upper limb amputees and 3 lower limb amputees. Conclusions: their quality of life, according to the central human functional capacities defined by Nussbaum, reflects that after the accident they were affected by the loss of security and self-confidence, preventing social activities. Their current condition limits them from improving their income due to their disabling condition. Chronic pain and prosthetic maladjustment are common, as well as the use of accessories and the difficulties to move. The strengthening of the primary family support network is evident but, at the end of their rehabilitation, there is a lack of interest in social activities. Participants have concrete future projects that involve their family. Finally, their rehabilitation process was limited to the basics and they did not have any other type of support.
\end{abstract}

\section{Key words}

Perception, amputation, work accident, quality of life, worker (Source: Mesh, NCBI).

\section{Percepção de qualidade de vida em trabalhadores vítimas de acidente laboral que acabou em amputação.}

\section{Resumo}

Objetivo: descrever a percepção da qualidade de vida das pessoas que têm sofrido amputação por um acidente laboral. Metodologia: pesquisa qualitativo com a aplicação de entrevista semiestruturadas de 10 perguntas abertas a seis trabalhadores que sofreram um acidente laboral (trabalho) que acabou em amputação. A informação foi coletada, analisada e classificada de forma manual em categorias emergentes. Resultados: os participantes são de sexo masculino, de 34 e 52 anos de idade, seu domicílio são os municípios do eixo Cafeeiro e norte do departamento do Valle (Colômbia), o estado civil é 5 casados e 1 separado, o nível educativo 2 no ensino primário, 3 com ensino médio e 1 técnico profissional. Todos cadastrados no sistema de saúde. Os segmentos corpóreos amputados são: 3 amputados do Membro Superior, os outros três amputados do Membro Inferior. Conclusões: De acordo com a lista das capacidades humanas de Nussbaum evidencia-se que, após do sinistro, a qualidade de vida das pessoas amputadas foi afetada na perda de segurança e de confiança dos indivíduos em si mesmos, o qual os impede de realizarem atividades de tipo social. Sua condição atual de deficiência limita sua capacidade de melhorar seus ingressos, sendo a dor crónica e a desadaptação protésica fatores comuns, junto com o uso de aditamentos e as dificuldades na locomoção. É evidente o fortalecimento da rede de apoio familiar primária; entretanto, ao finalizar sua reabilitação há desinteresse em atividades sociais. Os participantes têm projetos concretos envolvendo sua família. Finalmente, seu processo de reabilitação se limitou a uma atenção básica e não contaram com outro tipo de apoio.

\section{Palavras Chave}

Percepção, amputação, acidente de trabalho, qualidade de vida, trabalhador (Fonte: DeCS, BIREME). 


\section{Introducción}

Durante el 2017 se presentaron diariamente 1.800 accidentes laborales. Aunque el número reportado disminuyó en un $6.5 \%$ en relación con 2016 , el costo en el que incurrieron las Administradoras de Riesgos Laborales (ARL) para atenderlos aumentó un 12,1\% real anual (1). Los sectores que reportan mayor accidentalidad en Colombia son los relacionados con actividades inmobiliarias, industria de manufacturas, construcción y comercio. La Federación de Aseguradores Colombianos (FASECOLDA) mencionan que Bogotá, con 188.015 casos (28.7\%), y Antioquia, con 137.688 casos (21\%), de 655.570 total de casos, son las regiones que presentan mayor número de accidentes laborales reportados en 2016 (1).

Según la gerente técnico-médica de Colmena Seguros, "la inseguridad vial, relacionada con motos, está contribuyendo cada vez más a estos eventos"(2), debido, no solo por la actividad de la mensajería, sino cuando los trabajadores en cumplimiento de sus funciones, como visitas a clientes, proveedores $\mathrm{u}$ otras empresas, son víctimas de todo tipo de accidentes, incluyendo los de tránsito y la siniestralidad en las ARL alcanza el $60 \%$, un nivel que se mantiene sin mayores variaciones frente a años anteriores, a pesar de que el número de accidentes ha caído (2). Muchos de estos accidentes laborales terminaron en la amputación de algún miembro, afectando la calidad de vida de estos trabajadores. Posterior a ese suceso y de la pérdida física, las instituciones responsables de estos trabajadores deben brindarle toda la rehabilitación que requieren para de alguna manera reintegrarlo a su vida tanto laboral como social.

La calidad de vida es considerada como una dimensión importante en el ser humano; abarca múltiples áreas que están relacionadas con el bienestar físico, bienestar social, la alimentación, las relaciones interpersonales y con otras especies, los proyectos y/o metas a corto, mediano o largo plazo. Todas estas áreas hacen parte de la gran dimensión calidad de vida (3). Según Martha Nussbaum, filósofa estadounidense quien será nuestra referente metodológica, existen 10 categorías de calidad de vida, las cuales son las siguientes: vida, salud corporal, integridad corporal, sentidos, imaginación y pensamiento, emociones, razón práctica, afiliación, otras especies, capacidad para jugar y control sobre el propio entorno (4). Sin embargo, la calidad de vida puede verse afectada por diferentes situaciones o eventos que interfieren algunas o todas de estas categorías, como sería el caso de un accidente laboral que terminó en amputación; este suceso puede afectar negativamente las categorías de calidad de vida resultando en afecciones en la vida de la persona.

Un accidente laboral que terminó en amputación es un suceso que afecta las diferentes áreas del ser humano, produciendo cambios en la vida de las personas, es por esto que, a través de este estudio de tipo fenomenológico, se analizaron las diferentes categorías de calidad de vida definidas por Marta Nussbaum, se busca describir la percepción de calidad de vida que tienen los trabajadores que han sufrido un accidente laboral que terminó en amputación. Para esto se realizaron 6 entrevistas semiestructuradas a los 6 participantes del estudio. La Guía de la entrevista incluyó preguntas relacionadas con las categorías definidas por Nussbaum para identificar cómo cada uno de ellos las percibe.

\section{Materioles y Método}

Estudio cualitativo fenomenológico descriptivo que busca comprender los fenómenos humanos a través de la descripción de la experiencia vivida, configurando unidades de significado y categorías comprensivas (5). La investigación incluyó 6 participantes que se obtuvieron por saturación de la información en las entrevistas. Ellos tuvieron un proceso de rehabilitación posterior al accidente laboral que terminó en amputación, en la ciudad 
de Pereira Risaralda. Fueron elegidos por ser consultantes de un servicio de medicina laboral. Los criterios de inclusión fueron: (a) Personas mayores de 18 años; (b) Haber tenido un proceso de rehabilitación posterior al accidente laboral que terminó en amputación. (c) Asistir a consulta de medicina laboral en la ciudad de Pereira Risaralda.

Este estudio tuvo en cuenta los principios éticos para las investigaciones en seres humanos, contenidas en la declaración del Helsinki como son "resguardar la intimidad de la persona que participa en la investigación y la confidencialidad de su información personal, para lo cual los participantes firmaron consentimiento informado, teniendo en cuenta las consideraciones reglamentadas para su firma (6).

La recolección de información se realizó en los meses de abril y mayo de 2019 mediante entrevistas semiestructuradas que fueron grabadas, transcritas palabra por palabra para su posterior análisis y fueron realizadas en el consultorio médico del servicio de medicina laboral donde consultaron.

Inicialmente el equipo de investigación describió el fenómeno de interés, declarando los supuestos de los investigadores; se realizaron las entrevistas, se transcribieron, se leyeron atentamente para descubrir el contenido y comprender las relaciones esenciales. La entrevista contó de 10 preguntas la cuales hacía alusión a las categorías de calidad de vida definidas por Martha Nussbaum:

1. ¿Cómo describe su vida antes y después del accidente laboral y como es su vivienda, el barrio donde vive, con quien vive, cómo es su vida social y sus ingresos?

2. ¿Cómo escribe su condición actual de saludy su alimentación?

3. ¿Cómo se desplaza normalmente, lo hace libremente, sin límites, de un lugar a otro?

4. ¿Usted está en capacidad y libertad de utilizar su imaginación y su pensamiento en asuntos como la religión, la lectura o las artes?
5. ¿Cómo son las relaciones afectivas de las personas más cercanas a usted, como su pareja, sus hijos, sus padres o hermanos?

6. ¿Qué aspectos positivos puede describir de usted mismo y de sus condiciones de vida y que otros considera que podría mejorar?

7. ¿Qué opina sobre el trato recibido por usted de los demás en relación a la igualdad de los derechos, pues por la discapacidad que usted tiene?

8. ¿Vive usted con mascotas o animales domésticos y como asegura su cuidado y bienestar?

9. ¿En qué grupos o actividades recreativas, deportivas, artísticas o culturales participa?

10. ¿Qué planes tiene y como se ve en el futuro?

El tiempo aproximado de duración de cada entrevista fue de 20 minutos, fueron grabadas, transcritas en un archivo Microsoft Word, para posteriormente ser analizados a través del método de categorización y triangulación. Esta triangulación se realizó a través del uso de la estrategia de entrevistas individuales en donde se analizó la información obtenida sobre el fenómeno que queríamos estudiar: la percepción de la calidad de vida posterior a un accidente laboral que terminó en amputación. Aunque los pacientes se contactaron a través de una consulta médica, no se realizó revisión de historia clínica ni de datos confidenciales, tampoco se relacionaron con la institución a la que consultaban.

\section{Resultados}

Todos los participantes incluidos en el estudio son de sexo masculino que se encontraban entre los 34 y 52 años de edad; el sitio del domicilio de estos está ubicado en municipios del eje cafetero y norte del valle como Dosquebradas, Cartago, Pereira, Santa Rosa de Cabal y Viterbo; el estado civil es 5 casados y 1 separado; el nivel educativo 2 en primaria, 3 con nivel de secundaria y 1 técnico profesional; 3 de los participantes están afiliados a Medimás, 2 a Salud Total y 1 a SOS. 
Respecto a los segmentos corporales amputados de la población participante, 3 de los participantes fueron amputados del Miembro Superior así: 1 del Miembro Superior Izquierdo, 1 de la mano derecha y 1 de la mano izquierda. Los otros 3 fueron amputados del Miembro Inferior así: 1 de todo el Miembro Inferior Derecho, 1 de pierna izquierda y 1 de tercio distal de la pierna derecha.

Los resultados obtenidos en este estudio arrojaron una serie de variables que generaron en los autores la definición de categorías emergentes de las cuales se puede describir lo siguiente: todos los participantes consideraron que contaban con una vida funcional antes del siniestro, gozaban una vida muy activa en la cual realizaban diferentes actividades de tipo social, recreativo, entre otras; se considera por lo tanto que todos los entrevistados realizaban sin obstáculos o inconvenientes, todas las actividades de la vida diario. Todos manifestaron que, posterior a la amputación de su segmento corporal afectado, desencadenó en una gran pérdida funcional que los llevó a dejar de realizar múltiples actividades que hacían previamente. Sus ingresos disminuyeron considerablemente, pues 5 de los entrevistados fueron pensionados por sus ARL recibiendo mensualmente un salario mínimo mensual sin acceso a otro tipo de ingreso como consecuencia de su discapacidad. A pesar de que el accidente laboral les cambió la vida, este no afectó su alimentación, pues se ha mantenido de manera adecuada y sin ninguna modificación. La amputación les ha generado un dolor crónico y permanente, y el uso de la prótesis ha exacerbado esta condición al punto de que uno de ellos nunca pudo utilizarla. Para los traslados y desplazamientos requieren de ayudas técnicas externas para realizarlos como bastones, muletas y vehículo adecuados a su discapacidad.

Respecto a las actividades de tipo religioso o artístico, éstas no se han visto afectadas, consideran que tienen libre elección para realizarlas, aunque se limitan prácticamente solo a las de tipo religioso, las actividades artísticas, aunque tienen la libre elección de hacerlas, ninguno las practica. Las relaciones familiares se vieron fortalecidas después del siniestro, contaron con el apoyo de su núcleo familiar primario y en especial de su pareja; a excepción de uno de los participantes que terminó con la separación de su cónyuge. Los entrevistados manifestaron que con el transcurrir de los días ciertas actividades familiares como reuniones o celebraciones perdieron el interés que para ellos usualmente tenían antes del siniestro, lo que afectó considerablemente la asistencia de ellos a las mismas. En cuanto al desarrollo personal y los proyectos a futuro, los entrevistados muestran que sus deseos son tener una vida tranquila, dan prioridad a sacar sus hijos adelante y a dos de ellos les gustaría vivir en el exterior. Todos manifestaron que el apoyo familiar fue suficiente, se mantuvo una red de ayuda cercana que les permitió superar todo el proceso de rehabilitación. La percepción de desigualdad se sustenta en el cumplimiento mínimo por parte de la aseguradora de las prestaciones asistenciales y económicas, dejando de lado aspectos tan importantes como la inclusión de la familia y la integración social.

Respecto a la relación con otras especies, ellos manifiestan que todos tienen la posibilidad de tener mascotas y asegurar su cuidado, todos tienen mascotas a excepción de uno que manifestó no gustarles. Una de las actividades de la vida que se vio afectada en todos los participantes son las actividades de tipo recreativo y deportivo, ninguno la practica en la actualidad, manifestaron que no tienen el interés de hacerlas, a pesar de que antes practicaban algún tipo de actividad deportiva o recreativa. Finalmente, con relación a las metas personales, los trabajadores manifestaron todos tener metas a futuro, la mayoría de estas se centraron en tener una vida tranquila y obtener mayores ingresos económicos, siempre al lado de sus familias.

Acontinuación, se definen las categorías deductivas referenciadas por Nussbaum y las categorías emergentes determinadas por los investigadores. 


\section{Categoría deductiva: VIDA}

Categorías emergentes determinadas por los investigadores:

\section{Persona Previamente Funcional}

Término que incluye funciones corporales, actividades y participación. Indica aspectos positivos de la interacción entre una persona, con una determinada condición de salud y su entorno (5). Cada uno de los entrevistados manifiesta que su vida antes de la ocurrencia del accidente era una vida normal, sin inconvenientes, en la cual desarrollaban actividades recreativas, con proyectos, sueños, eran personas activas, llevaban una vida normal sin ningún tipo de restricciones.

\begin{abstract}
"Bueno, mi vida antes del accidente, pues, lo que normalmente hace un joven, yo cuando tuve el accidente tenía 27 años de edad, era un joven, pues no sé, lo soy, de mucho ambiente, muy, una persona con mucho carisma, me rio por todo, siempre he sido una persona positiva, trabajador, trabajo desde que terminé el colegio, entonces, no, siempre he sido una persona echada para adelante, trabajador, eso antes del accidente, después del accidente, perdí, les voy a decir la verdad perdí un poco de seguridad en mí, yo era un persona muy segura de las cosas que hacía, perdí un poco de seguridad, porque, porque pues la pérdida de un miembro, o una parte del cuerpo de uno, lo hace que uno pierda esa confianza que uno siempre ha tenido, pero igualmente sigo siendo la persona risueña, la persona de empuje, y la misma persona, la misma persona, me hace, perdí un poco de seguridad en mí, pero en mi esencia sigo siendo la misma persona"
\end{abstract}

\section{Menos funcional}

Cambio en los comportamientos que puede realizarse con el suficiente automatismo o cambio en las acciones que exigen dedicarles tiempo y energía (7). Los entrevistados manifiestan que posterior al accidente la vida les cambio mucho, manifiestan que han perdido la seguridad en sí mismos, la confianza, ya no volvieron a realizar los planes que hacía antes del accidente, sumado a esto, los dolores que les ha dejado han afectado tanto su vida emocional como social.

\section{Menos Ingresos}

Disminución de los recursos de un individuo ya sea vía salarios, inversiones u otros (8). Los ingresos de la mayoría de los entrevistados corresponden a un salario mínimo por pensión de invalidez; uno de ellos aún continúa trabajando y otro se encuentra desempleado. Su condición de pensionados por invalidez es una limitante para poder mejorar sus ingresos salariales en estos momentos.

\section{Categoría deductiva: SALUD CORPORAL}

Categorías emergentes determinadas por los investigadores:

\section{Adecuada alimentación}

Es una dieta que cumple con las siguientes características: completa, equilibrada inocua, variada y adecuada. Alimentación que debe estar de acuerdo con la edad del comensal, la actividad física y sus costumbres (7). Todos los entrevistados manifiestan que el accidente sufrido no ha interferido en nada su alimentación, ella sigue siendo normal, adecuada y suficiente.

\section{Dolor crónico y dificultad con la prótesis}

Dolor que se extiende por más de 3 a 6 meses desde su inicio o que va más allá del período de curación de una patología, o se asocia a una enfermedad crónica (9). Los entrevistados manifiestan que mantienen con un dolor permanente en el sitio de la amputación relacionado con la poca adaptación a la prótesis que usan, ésta les ha generado múltiples dolencias y uno de ellos no la toleró. 
"Pues mi condición actual, pues en este momento es muy pésima pues porque primero yo no sentía dolores de ninguna clase, ahora estos dolores son prácticamente mi diario porque mantienen ahí, con medicamentos no hay forma de que al menos apacigüe el dolor que yo diga ay Dios hoy no sentí dolor, siempre he mantenido el dolor ahí pues ahora últimamente que me han salido como unos corrientazos que me dan y me deja como muy este el brazo y todo eso pero pues ahí vamos"

\section{Categoría deductiva: INTEGRIDAD CORPORAL}

Categoría emergente determinada por los investigadores:

\section{Dificultades y adecuaciones para el traslado}

Tomar las medidas necesarias cuando se presenten dificultades para andar y desplazarse por el entorno. Avanzar sobre una superficie a pie, paso a paso, de manera que al menos un pie esté siempre en el suelo, como caminar hacia delante, hacia atrás o de lado. Mover todo el cuerpo de un sitio a otro de una manera que no sea andando, como escalar una roca, correr por una calle, saltar en un pie, corretear, brincar, correr esquivan obstáculos (5). Los entrevistados todos han tenido que hacer adecuaciones y cambios para el traslado de un lugar a otro; como por ejemplo utilizar motocicletas apropiadas para su discapacidad, ortesis como bastones y cuidados relacionados con el uso del transporte público en bus.

"Si porque si uno se va a montar a la buseta, la gente le pregunta a uno, que te pasó, como esto, pues yo soy una persona que soy sociable, yo le digo a la gente que fue lo que me paso, para que, yo a la gente le digo no, cuiden su cuerpo, cuiden su manito, que mire que esto y esto, o bueno parte de su cuerpo; y la gente me dice no, duro, como sería el dolor, y si es un dolor muy grande no tener mano pero de todas maneras Dios en muy bello con uno, todo en manos de Dios"

\section{Categoría deductiva: SENTIDOS, IMAGINACIÓN Y PENSAMIENTO:}

Categoría emergente determinada por los investigadores:

\section{Libertad de elección}

Describe la oportunidad y la autonomía de un individuo para realizar una acción seleccionada de al menos dos opciones disponibles, sin restricciones por parte de terceros. Todos los entrevistados se sienten y tienen la libertad de profesar la religión que desean y de participar y realizar actividades como la lectura y las artes. Sin embargo, hay desinterés por las mismas.

"No, la católica, ¿no lee nada? No, pues en el pueblo no hay cine, no nada"

\section{Categoría deductiva: EMOCIONES}

Categorías emergentes determinadas por los investigadores:

Fortalecimiento de las relaciones familiares y de pareja

Mejorar las relaciones de la unidad social, compuesta por un grupo de personas unidas por vínculos de consanguinidad, afectivos o de vivienda, y que a los efectos de las normas sociales vigentes deben proponerse ayuda mutua, compartir recursos, comunicarse entre sí, procurar el bien propio, así como contribuir al de su comunidad (10). Las relaciones familiares y de pareja de los entrevistados se vieron fortalecidas a raíz de los 
accidentes laborales. Todos han sentido el apoyo familiar más de la familia cercana lo que les ha permitido confrontar el siniestro.

\section{Afectación familiar}

Influencia en la unidad social, compuesta por un grupo de personas unidas por vínculos de consanguinidad, afectivos y de vivienda, y que según las normas sociales deben procurarse ayuda mutua, compartir los recursos, comunicarse entre sí, procurarse el bien propio, así como contribuir al de su comunidad (9). La respuesta inicial de los entrevistados hacia sus familias fue de agresividad relacionada con el proceso de adaptación a la pérdida y posterior a la rehabilitación se observa aislamiento y poca participación en las actividades familiares.

\begin{abstract}
"¿Cómo vivo con ella? Pues en el momento de que, de que tuve el accidente pues ella ha estado muy pendiente de mí, pues siempre yo soy el que de pronto a veces mantengo malgeniado por lo que me paso el accidente y todo, pues ella me ha de comprender pues con mucho dolor y mucha dificultad pues siempre con la prótesis y todo, pues yo le digo que me comprenda porque a veces para no tener pues como problemas, más sin embargo siempre así tiene uno sus, sus roces de problema con el hogar cierto"
\end{abstract}

\section{Categoría deductiva: RAZÓN PRÁCTICA}

Categoría emergente identificadas por los investigadores:

\section{Desarrollo Personal y proyectos a futuro}

Incluye actividades que mejoran la conciencia y la identidad, impulsan el desarrollo de las habilidades personales y de los propios potenciales, contribuyen a construir capital humano y facilitan la empleabilidad, mejoran la calidad de vida y contribuyen a la realización de sueños y aspiraciones (11). Todos los participantes en general en sus proyectos a futuro tienen la idea de "estar tranquilos" y lo relacionan con asuntos como tener una vivienda propia, estar con su pareja y sus hijos y tener las posibilidades económicas y el tiempo para "sacar sus hijos adelante". Dos de ellos se imaginan viviendo en el exterior.

"Bueno, aspectos míos positivos como ya les había dicho, soy muy amigable, soy mucho de hablar, me gusta ayudarle a las personas cuando puedo ayudarles, me gusta escuchar a las personas, soy bueno escuchando, bueno dando consejos, trato de que las personas se sientan, se sientan bien al lado mío, soy una persona alegre"

\section{Categoría deductiva: AFILIACIÓN}

Categorías emergentes identificadas por los investigadores:

\section{Apoyo familiar}

Soporte de la unidad social, compuesta por un grupo de personas unidas por vínculos consanguíneos, afectivos y de vivienda y que según las normas sociales vigentes deben procurarse ayuda mutua, compartir los recursos, comunicarse entre sí, procurarse el bien propio, así como contribuir al de su comunidad (9). Todos los participantes manifiestan que tuvieron una adecuada red de apoyo familiar en los momentos posteriores al siniestro ya sea la familia cercana o lejana. No percibieron de ninguno de ellos alejamiento o rechazo.

\section{Percepción de desigualdad y aislamiento familiar}

Apreciación de disconformidad entre lo que tiene derecho y no lo obtiene. El aislamiento social es cuando una persona se aísla del resto de la sociedad o siente que está aislada. Esto suele ocurrir en personas con carácter problemático o con incapacidad para hacer amigos por motivos 
tan comunes como: timidez o excesiva timidez (12). A pesar de contar con la red de apoyo familiar y percibir que se cumplieron sus expectativas asistenciales y prestacionales por parte del sistema general de riesgos laborales, todos manifiestan que solo han recibido el tratamiento básico y mínimo, sin haberles brindado otro tipo de apoyo por parte del gobierno u otro tipo de instituciones.

"Con mis otros familiares pues bien, no me rechazan que por ser amputado, no sigue uno lo mismo, no, no normal, gracias a Dios normal"

\section{Categoría deductiva: OTRAS ESPECIES}

Categoría emergente identificada por los investigadores:

\section{Posibilidad de relación con otras especies}

Se trata específicamente de animales compañeros, son aquellos animales que colaboran con el hombre o conviven con él. Gracias a esto pueden ser denominados "animales domésticos"; la característica es que con tales animales es posible una comunicación, ya que el hombre se convierte para ellos en un posible compañero social (13). Todos los participantes tienen las capacidades físicas y económicas de relacionarse con otras especies, en este caso animales de compañía, y de hecho todos cuentan con una o varias mascotas que consideran miembros de su familia, a excepción de uno que manifiesta no gustarle las mascotas.

"Bueno, en la casa somos mi esposa, mi hermano, un perrito que se llama Tego y mi persona, lo queremos muchos, si tenemos un perrito, es un criollo, un perrito criollo, pues como aseguramos, no, no mi hermano es el prácticamente el que se encarga de él pues igual mi esposa y yo le damos, lo alimentamos, y le compramos su comida, su alimento, pero el que más se encarga de él, nosotros le damos, como se dice ese cariño de hogar pero el que más se encarga, el que le hace los alimentos es mi hermano"

\section{Categoría deductiva: CAPACIDAD PARA JUGAR}

Categoría emergente identificada por los investigadores:

\section{Desinterés por la recreación y el deporte}

Desapego y desprendimiento de todo provecho personal, próximo o remoto hacia el deporte que es una actividad reglamentada, normalmente de carácter competitivo, que puede mejorar la condición física y a la recreación, que es la actividad destinada al aprovechamiento del tiempo libre para el esparcimiento físico y mental (14). Ninguno de los participantes manifiesta el interés de participar en actividades de ocio y recreación; a pesar de que todos antes del accidente realizaban alguna actividad de tipo deportivo y recreativo, en la actualidad no la practican.

\begin{abstract}
"No, en el momento no estoy en ninguna, en ninguna entidad no, voy, voy cuando puedo a piscina me encanta hacer, pues el deporte que practico ahora después del accidente por decirlo así, me gusta mucho la natación, hace tiempo estuve en la Villa haciendo natación y ahora voy esporádicamente, voy a piscina y nado, ese es el deporte que hago y caminar"
\end{abstract}

\section{Categoría deductiva: CONTROL SOBRE ENTORNO DE CADA UNO}

Categoría emergente identificada por los investigadores:

\section{Metas personales}

Una meta es un resultado deseado que una persona o un sistema imagina, planea y se compromete a lograr, un punto final deseado personalmente en una organización en algún desarrollo asumido. 
Muchas personas tratan de alcanzar objetivos dentro de un tiempo finito, fijando plazos (15). Todos los entrevistados tienen "sueños" planes y metas a corto y largo plazo, y que está relacionado con lograr una vida tranquila en compañía de su familia y mejorando sus condiciones de vida e ingreso incluso en otros países.

\begin{abstract}
"Bueno en el futuro inmediato me veo con mi esposa y un hijo, no tenemos hijos, me gustaría tener un hijo, por ahí estuve pues tratando o queriendo entrar a estudiar, sino que se me hace un poco difícil por, por lo monetario, por decirlo así, pero pues me gustaría estudiar regencia en farmacia que siempre quise estudiar regencia en farmacia, por lo que pues yo ya tengo conocimientos en cuestiones de la salud, trabajé en Saludcoop, en la Clínica San Rafael y pues me gustaría estudiar regencia en farmacia y pues inmediato, inmediato, me gustaría, no sé, tener un hijo, me veo con una familia más grande, más amplia en mi futuro"
\end{abstract}

\section{Discusión}

En Colombia, se encontró que se han realizado estudios de tipo cuantitativo relacionados con calidad de vida en trabajadores que han sufrido un accidente laboral, uno de ellos es el estudio calidad de vida de las personas que han sufrido accidente laboral atendidos en el Hospital General de Medellín el año 2012 (15), los resultados de este estudio exponen que no se vio afectada la calidad de vida de estos trabajadores, la calificación obtenida fue superior a la media, es decir, demostró óptimas condiciones de su calidad de vida, también refiere que solo el $20 \%$ de los trabajadores participantes presentaron algún tipo de inconvenientes posteriores al accidente laboral $\mathrm{y}$, en relación a la salud mental, el $85 \%$ de los participantes no presentó ningún tipo de alteración, demostraron tener buenas condiciones en el estado mental.
Así mismo, el estudio de Auto-percepción de la calidad de vida en pacientes con amputación de extremidad inferior, realizado en el Hospital Roosevlt en ciudad de Guatemala en el año 2015, aunque es un estudio de tipo cuantitativo, en el resultado del indicador media de la calidad de vida, el $75 \%$ de los participantes obtuvo un resultado de Calidad de Vida Aceptable o Alta; respecto al dominio físico, en este mismo estudio el resultado fue del $69 \%$ con las misma calificación, el dominio psicológico el $86 \%$ también obtuvo esta calificación, el ambiente y el entorno obtuvieron igual la misma calificación: el 85\% (16).

Estos estudios, aunque son de tipo cuantitativo reflejan que los pacientes no han tenido afectación en su calidad de vida, por lo tanto, les ha permitido seguir con pocos o ningún inconveniente, aspecto que en este estudio se confirma en los participantes, ya que sus resultados reflejan una afectación menor en dicha calidad de vida, aspectos que en este estudio cualitativo también se confirman.

El estudio denominado Análisis de la Calidad de Vida y Dolor en paciente amputados del 2017, también de tipo cuantitativo, utilizó el cuestionario de calidad de vida SF-36 que consta de 36 preguntas, valora los estados positivos y negativos de la salud y cubre 8 dimensiones, se realizó con 28 participantes amputados, los resultados fueron que la mitad de la muestra tuvieron una puntuación menor de 50 en el cuestionario, por lo que se catalogan como "peor calidad de vida o peor estado de salud", la otra mitad aparecen catalogadas como "mejor calidad de vida o mejor estado de salud". En la dimensión física, el $60.71 \%$ con mal rol físico y el $71 \%$ con mala salud mental. En cuanto a la función social y el rol emocional, el $60 \%$ de la muestra fue positiva. En cuanto al dolor, la mitad consideró no tener dolor y la otra mitad con dolor. Por lo anterior, lo reflejado en este estudio es que se encuentran divididas la percepción de la calidad de vida, en unos se afectan mientras que en los otros no (17). De igual manera la percepción de los participantes 
en este estudio muestra que hay afectación en su estado de salud relacionadas principalmente con dolor en el sitio de la amputación, sin embargo, en este estudio hay una gran afectación en el estado mental mientras que en nuestra investigación se demostró una afectación favorable posterior al siniestro. Finalmente, en ambos estudios se evidenció afectación en la función social.

El Instituto Nacional de Rehabilitación Pedro Aguirre Cerda realizó en el año 2016 en Chile un estudio cuantitativo de calidad de vida en salud en individuos amputados de extremidad inferior con prótesis; aplicó el cuestionario SF-12V12, los resultados arrojados evidencian mejoría en la calidad de vida, cuatro de los participantes del estudio expresaron que presentaban dolor por sobrecarga y otros cuatro manifestaron dolor al utilizar la prótesis coincidencias con nuestro estudio con respecto al dolor al usar la prótesis (18).

Desde la óptica fenomenológica, un estudio (19) en el cual se entrevistaron 52 personas que habían sufrido una amputación reflejó que los pacientes mostraron que vivir una amputación es triste, difícil, doloroso, pero a pesar de todas las dificultades y sufrimientos, no se dejan vencer. La expectativa de una vida nueva es motivo de felicidad y deseo de continuar seguir viviendo; estos resultados son similares con nuestro estudio en el cual también los participantes manifestaron que, a pesar de la pérdida de su segmento corporal, tienen aún sueños, proyectos por cumplir junto a su familia.

En el estudio calidad de vida de los pacientes amputados de la extremidad inferior, realizado con 65 pacientes (19), arrojaron resultados en la variable de vida cotidiana: el $40 \%$ de los participantes se trasladan de un lugar a otro de manera independiente; además el $85 \%$ de los pacientes dicen que cuentan con apoyo familiar y $15 \%$ expresaron que no. También estos resultados son coincidentes con los arrojados en nuestro estudio, porque demuestran la afectación mínima de la calidad de vida relacionada con el apoyo familiar, especialmente de sus cónyuges, a excepción de un participante de nuestro estudio que terminó en la separación de su pareja, lo que coincide con el $15 \%$ que expresaron que no tenían dicho apoyo.

Al parecer, en los pocos estudios realizados con respecto a la calidad de vida en pacientes amputados, la mayoría de los resultados demuestran que esta no se vio afectada, solo algunos pocos aspectos como el dolor crónico que les generó la amputación y el uso de la prótesis fueron los aspectos negativos que se reflejaron en los resultados igual que en nuestro estudio. Por esto es importante la realización de estudios mixtos para obtener una perspectiva más amplia y profunda del fenómeno, que resulte más integral, completa y holística, con el fin de producir datos más ricos y variados mediante la multiplicidad de observaciones, ya que pueden considerarse diversas fuentes, tipos de datos, contextos, ambientes y análisis.

Una teoría económica se concibió en la década de 1980 como un enfoque alternativo a la economía del bienestar. En este enfoque, Amartya Sen reúne una serie de ideas que anteriormente estaban excluidas de (o inadecuadamente formuladas en) los enfoques tradicionales a la economía del bienestar. El objetivo principal del enfoque de las capacidades es en lo que los individuos son capaces de hacer.

\section{Conclusiones}

La calidad de vida de los participantes del estudio con base en las capacidades funcionales humanas centrales definidas por Nussbaum, reflejan que la vida posterior a al siniestro se vio afectada en la pérdida de la seguridad y la confianza en sí mismos, impidiendo la realización de actividades que realizaban previamente como participación en reuniones familiares y de tipo social. Su 
condición actual de trabajadores, pensionados o desempleados con un salario mínimo los limita en la consecución de mejores ingresos debido a su condición de discapacidad.

El dolor crónico y la desadaptación protésica es un estado común en todos los participantes. El uso de aditamentos y las dificultades en el traslado, en especial al utilizar el transporte público, son condiciones generalizadas para los participantes.

Es evidente el fortalecimiento de la red de apoyo familiar primaria en especial del cónyuge, sin embargo, al finalizar los procesos de rehabilitación se evidencia desinterés en actividades recreativas, culturales y familiares que eran frecuentes antes del accidente.

A pesar del siniestro se evidencia que todos los participantes tienes proyectos a futuro concretos que involucran todo su núcleo familiar. Los trabajadores en su proceso de rehabilitación solo obtuvieron el tratamiento básico y no contaron con otro tipo de apoyo como del gobierno, otras fundaciones u organizaciones no gubernamentales.

\section{Agradecimientos}

Los autores expresan sus agradecimientos a los 6 participantes de la investigación quienes con sus emotivos testimonios hicieron posible la realización de este estudio; agradecemos también a nuestra asesora de investigación la Doctora Martha Cecilia Veloza por todo su acompañamiento y enseñanzas, y al Doctor Daniel Eslava por su excelente gestión como director de la Maestría Salud Pública y Desarrollo Social de la Fundación Universitaria del Área Andina de la ciudad de Bogotá. 


\section{Referencios bibliogróficos}

1. Garcia CA. Panorama de los accidentes de trabajo en Colombia [Internet]. El Tiempo. Bogotá: 2018 mzo. 3. Sección economìa [consultado 2019 abr.]. Disponible en: https://www.eltiempo.com/ economia/sectores/panorama-de-los-accidentes-de-trabajo-en-colombia-en-2017-189464

2. Colmena Seguros [Internet]. Colmena Seguros. Sección Noticias [consultado 2019 my.]. Disponible en: https://www.colmenaseguros.com/noticias-colmena/Paginas/noticias.aspx?NoticiaID=158

3. Hernández-Sampieri R, Fernández-Collado C, Baptista LP, editores. Metodología de la investigación. $6^{\mathrm{a}}$ ed. México D.F.: McGraw-Hill; 2014. 656 p.

4. Osorio-Alcalde JC. Libro: La calidad de vida. Reseña. Rev. Eleuthera [Internet]. 2016; 14: 129-132. Disponible en: http://eleuthera.ucaldas.edu.co/downloads/Eleuthera14_8.pdf

5. Asociación Médica Mundial. Declaración de Helsinki de la AMM-Principios éticos para las investigaciones médicas en seres humanos [Internet]. Centro de Documentación en Bioética; 2013 Dic. [Consultado 2019 my.]. Disponible en: http://www.redsamid.net/archivos/201606/2013declaracion-helsinki-brasil.pdf?1

6. Decreto 1507 de 2014. Manual Único para la Calificación de la pérdida laboral y ocupacional [Internet]. Bogotá: Ministerio del Trabajo; 2014. Disponible en: https://www.minsalud.gov.co/sites/ rid/Lists/BibliotecaDigital/RIDE/DE/DIJ/Decreto-1507-DE-2014.pdf

7. Enciclopedia Financiera [Internet]. [Consultado 2019 my.]. Disponible en: https://www. enciclopediafinanciera.com/about.htm

8. Instituto Colombiano de Bienestar Familiar. Guías alimentarias basadas en los alimentos para la población colombiana mayor de 2 años [Internet]. Ministerio de Salud: Bogotá; 2015 [Consultado 2019 jun.]. Disponible en: https:/www.minsalud.gov.co/sites/rid/Lists/BibliotecaDigital/RIDE/ $\mathrm{VS} / \mathrm{PP} / \mathrm{SNA} /$ guias-alimentarias-basadas-en-alimentos.pdf

9. Mesas-Idáñez A. Dolor agudo y crónico: clasificación del dolor [Internet]. 2012. Disponible en: https://www.academia.cat/files/425-11062-DOCUMENT/DolorAgutICronic.pdf

10. Centro de Estudios Demográficos (Universidad de la Habana). Diversidad y complejidad familiar en Cuba; 1999

11. Aubrey B. Managing your aspirations: developing personal enterprise in the global workplace. McGrawHill; 2010, 192 p.

12. Domenec BF. Programa de Habilidades Sociales. VS. 2012. Volumen 3. Página 17 a 23

13. Wolf U. Leben mit Tieren. Die Hauptformen und ihre ethischen Implikationen en ZDF-Nachtstudio, ed., Mensch und Tier, Geschichte einer heiklen Beziehung, Frankfurt; 2001. pp 40-59. http://www. altex.ch/resources/altex_2002_4_163_191_Teutsch.pdf

14. Diccionario de la Lengua Castellana (Real Academia Española). Diccionario [Internet]; 2014 [Consultado 2019 jun.]. Disponible en https://dle.rae.es/?id=DgIqVCc

15. Grant AM. An integrated model of goal-focused coaching: an evidence-based framework for teaching and practice. International Coaching Psichology. 2012; 7(2): 146-165. Disponible en: https://www.academia.edu/18048251/An_integrated_model_of_goal_focused_coaching

16. Colorado-Agudelo JD. Calidad de vida de las personas que han sufrido accidente laboral, atendidos en el Hospital General de Medellín en el año 2012 [Internet]. Medellín: Universidad CES; 2014. 118 p. Disponible en: file://C:/Users/lfy04/AppData/Local/Temp/MicrosoftEdgeDownloads/4d0dbe31eb95-4bc1-8360-958c9a11c038/calidad-de-vida-de-las-personas-que-han-sufrido-accidentelaboral.pdf 
17. Rodas-Jerez PA. Auto-percepción de la calidad de vida en pacientes con amputación de extremidad inferior [tesis de grado]. [Guatemala]: Hospital de Roosevelt; 2015 ag.

18. Jiménez-Rodríguez D, Ramírez-Peinado A, Rueda-Garrido JC, Dia-Agez JL. Análisis de la calidad de vida y el dolor en pacientes amputados. Revista de Psicología de la Salud. 2017; 5(1): Página 28 a 58

19. Cabrera-Rosales MJ. Calidad de vida en salud, en individuos amputados de extremidad inferior con prótesis del Instituto Nacional de Rehabilitación Pedro Aguirre Cerda [tesis de grado]. [Santiago de Chile]: Universidad de Chile; 2016 sept. 